\title{
The analysis and correction of transfer function of film measuring transducers of the microwave power
}

\author{
Igor Zakharov ${ }^{1,2}$, Pavel Neyezhmakov ${ }^{1}$ \\ ${ }^{1}$ National Scientific Centre "Institute of Metrology", 42, Myronosytska str., 61002, Kharkiv, Ukraine \\ ${ }^{2}$ Kharkiv National University of Radioelectronics, 14, Nauky ave., 61166, Kharkiv, Ukraine \\ E-mail (Igor Zakharov) newzip@ukr.net.
}

\begin{abstract}
Summary:
Results of determination of transient response of film thermoelectric measuring transducer of the microwave power are stated. On the basis of the analysis of their transfer function synthesis of the correctional device is made, allowing to reduce inertial properties of the transducer by three orders.
\end{abstract}

Keywords: film thermoelectric transducer, transient response, transfer function, correction, correctional device

\section{Introduction}

For measuring microwave power, film thermoelectric measuring transducers (FMT) are widely used. The advantages of FMT include high sensitivity, independence of readings from ambient temperature, and the ability to calibrate with low frequency alternating current. A considerable drawback of FMT is their significant inertia (15-20 ms), which prevents their use for dynamic measurements of microwave power. The problem of reducing the inertia of measuring transducers is most effectively solved by applying methods of electrical correction of their dynamic characteristics. To implement the corrective device, it is necessary to conduct a detailed analysis of the dynamic characteristics, which have a number of features.

\section{Transient response of FMT}

The experimental determination of the transient response (TR) of the microwave FMT was carried out on the basis of the method [1] of stepby-step measurement of TR by feeding power pulses with decreasing duration to the FMT input with an inversely proportional increase in their amplitude. This provided an increase in the signal-to-noise ratio during measurements in the initial sections of the TR. The instantaneous values of the FMT output signal were measured with a stroboscopic transducer. The processing of the measurement results was reduced to the normalization of TR in different areas. The identification of the normalized TP was carried out by the sequential logarithm method [2].

The identification of the FMT transient response showed that it is well described by the sum of 3

$$
\begin{aligned}
& \text { exponentials with positive coefficients } A_{i} \\
& \left(\sum_{i=1}^{3} A_{i}=1\right) \text { : } \\
& \qquad h(t)=1-\sum_{i=1}^{3} A_{i} e^{-\frac{t}{\tau_{i}}},
\end{aligned}
$$

where $\tau_{i}>\tau_{i+1}$ are the time constants of the FMT.

\section{Analysis of FMT transfer function}

The transition characteristic (1) corresponds to the transfer function

$$
\mathrm{H}_{\mathrm{FTP}}(\mathrm{s})=\sum_{\mathrm{i}=1}^{3} \frac{\mathrm{A}_{\mathrm{i}}}{\tau_{\mathrm{i}} \mathrm{s}+1} .
$$

As a result of studying the structure of the FTP transfer function, its following regularities were determined:

1) the FTP transfer function of the form (2) at has 3 real negative poles $-\frac{1}{\tau_{i}}$ and 2 real negative zeros $-\frac{1}{\alpha_{i}}$

2) the zeros of the FTP transfer function are located between its poles, and there is one zero between two adjacent poles:

$$
-\frac{1}{\alpha_{i}} \in\left(-\frac{1}{\tau_{i+1}} ;-\frac{1}{\tau_{i}}\right), i=0,1,2 . .
$$


Based on the revealed patterns, the transfer function of the FTP can be written in the following form:

$$
H_{\Pi}(s)=\frac{\left(\alpha_{1} s+1\right)\left(\alpha_{2} s+1\right)}{\left(\tau_{1} s+1\right)\left(\tau_{2} s+1\right)\left(\tau_{3} s+1\right)},
$$

where $\alpha_{i} \in\left(\tau_{\mathrm{i}-1} ; \tau_{i}\right)$.

The values $\alpha_{1}, \alpha_{2}$ can be found from the following system of equations:

$$
\left\{\begin{array}{c}
\alpha_{1} \alpha_{2}=A_{1} \tau_{2} \tau_{3}+A_{2} \tau_{1} \tau_{3}+A_{3} \tau_{1} \tau_{2} \\
\alpha_{1}+\alpha_{2}=A_{1}\left(\tau_{2}+\tau_{3}\right)+A_{2}\left(\tau_{1}+\tau_{3}\right)+A_{3}\left(\tau_{1}+\tau_{2}\right) .
\end{array}\right.
$$

\section{Correction device synthesis}

With sequential correction in the basis of the first-order aperiodic link with a time constant $\tau_{b}$, it is possible to write for the investigated FTP:

$$
H_{\mathrm{CD}}(s)=\frac{k_{b}}{\tau_{b} s+1} \cdot \frac{\left(\tau_{1} s+1\right)\left(\tau_{2}+1\right)\left(\tau_{3} s+1\right)}{\left(\alpha_{1} s+1\right)\left(\alpha_{2} s+1\right)}
$$

Assuming that $\tau_{b}=\tau_{3}$, we get:

$$
H_{\mathrm{CD}}(s)=k_{b} \frac{\left(\tau_{1} s+1\right)\left(\tau_{2} s+1\right)}{\left(\alpha_{1} s+1\right)\left(\alpha_{2} s+1\right)}
$$

Considering that $\alpha_{i} \in\left(\tau_{i-1} ; \tau_{i}\right)$ it can be concluded that the structure of the correcting device (CD) with a transfer characteristic of the form (5) is 2 sequentially connected simplest corrective links with a correction factor $k_{c i}=\frac{\tau_{i}}{\alpha_{i}}$

and a time constant $\tau_{i}$ [3].

Thus, the static conversion factor of the synthesized CD when it is implemented in the specified form on passive elements will be equal to

$$
k_{b}=\frac{\alpha_{1} \alpha_{2}}{\tau_{1} \tau_{2}}
$$

and the correction factor is determined by the expression

$$
k_{c}=\frac{\tau_{1}}{\tau_{3}}
$$

The coefficient of transformation of the CD is calculated by the formula

$$
k_{0}=\tau_{3} \sum_{i=1}^{3} \frac{A_{i}}{\tau_{i}}
$$

The value of the conversion coefficients can vary from $\frac{\tau_{3}}{\tau_{1}}$ (at $\mathrm{A}_{1}=1, \mathrm{~A}_{1}=\mathrm{A}_{2}=\mathrm{A}_{3}=0$, i.e., for a transducer without films) to 1 (at $A_{3}=1, A_{1}$
$=\mathrm{A}_{2}=0$ ). Therefore, for any positive $\mathrm{A}_{i}$, $k_{0}^{-1}<k_{c}$.

Thus, the correction of the FTP with the largest time constant $\tau_{1}$ in the basis of the first-order aperiodic link with a time constant $\tau_{n}$ with equal correction coefficients (the smallest time constant of the FTP) compared to the correction of the firstorder aperiodic transformer with a time constant $\tau_{1}$ with equal coefficients correction, gives a gain in the amplitude of the output signal in

$$
r_{n}=\tau_{3} \sum_{i=1}^{3} \frac{A_{i}}{\tau_{i}}
$$

time.

For the FTP investigated in subsection 1 of the article with the parameters indicated in Table 1, the gain in signal amplitude will be 20.4 times, i.e. with a decrease in the result of the correction of the FTP time constant by 1000 times, its output signal will decrease only by 49 times.

\section{Conclusion}

1. As a result of the study of the structure of the transfer function of the FTP, the following regularities were determined:

- the transfer function of the FTP has 3 real negative poles and a 2 real negative zeros;

- the zeros of the FTP transfer function are located between its poles,

- there is only one zero between two adjacent poles.

2. As a result of the implementation of the sequential correction, the model of the corrected FTP is the aperiodic link of the first order, which is equivalent to lowering the order of its differential equation to 1 . In this case, the time constant of the corrected FTP is equal to the smallest time constant of the uncorrected FTP, which is 3 less than its maximum time constant, and the amplitude of the output signal (and the signal-to-noise ratio) decreases only by 49 times.

\section{References}

[1] I.P. Zakharov. Method for determining the normalized transient response of a microwave power converter. A.C. SSSR No. 1651243, B.I. No. 19, 1991. (In Russian).

[2] I.P. Zakharov, M.P. Serhiienko. Determination of dynamic characteristics of measuring instruments. Kharkov, NURE, 2019, 210 p. (In Ukrainian).

[3] V.E. Shukshunov Corrective links in devices for measuring non-stationary temperatures. Moscow, Energiya, 1970, 120 p. (In Russian). 